\title{
COMPOSIÇÃO FLORÍSTICA E FITOSSOCIOLOGIA DE UMA ÁREA DE CAATINGA EM CONTENDAS DO SINCORÁ, BAHIA, MICRORREGIÃO HOMOGÊNEA DA CHAPADA DIAMANTINA
}

\author{
Paulo César Fernandes Lima ${ }^{1}$ \\ José Luciano Santos de Lima ${ }^{1}$
}

Recebido em 22/01/1999. Aceito em 01/07/1999

\begin{abstract}
RESUMO - (Composição florísitica e fitossociologia de uma área de caatinga em Contendas do Sincorá, Bahia, microrregião homogênea da Chapada Diamantina). Objetivou-se levantar a cobertura vegetal de 11.034 ha da Fazenda Extrema-Lapinha, 295 a $380 \mathrm{~m}$ de altitude $\left(13^{\circ} 46^{\prime}\right.$ a $14^{\circ} \mathrm{S}$ e $41^{\circ} 3^{\prime}$ a $\left.41^{\circ} 10^{\prime} \mathrm{W}\right)$, Contendas do Sincorá, BA, na Chapada Diamantina. O clima é do tipo BSwh', estépico, semi-árido quente, com precipitação anual de $500-700 \mathrm{~mm}$, período chuvoso de novembro a janeiro, temperatura média de $23^{\circ} \mathrm{C}$ e umidade relativa de $60-80 \%$. Predominam os solos Podzólicos VermelhoAmarelo Equivalente Eutrófico e solos Litólicos Eutróficos, em relevo levemente ondulado. Foram distribuídas aleatoriamente 100 parcelas de $20 \times 5 \mathrm{~m}$, sendo inventariadas as plantas com DAP $\geq$ a $5 \mathrm{~cm}$. Foram encontrados 2.897 indivíduos abrangendo 71 espécies, 51 gêneros e 23 famílias botânicas, não sendo identificados $3,59 \%$ dos mesmos. As famílias Euphorbiaceae $(15,67 \%)$, Mimosaceae $(13,80 \%)$, Bignoniaceae $(12,35 \%)$, Caesalpiniaceae $(7,14 \%)$, Boraginaceae $(6,63 \%)$, Polygonaceae $(6,63 \%)$, Anacardiaceae $(6,04 \%)$, Burseraceae $(4,93 \%)$, Apocynaceae $(4,59 \%)$ e Combretaceae $(4,28 \%)$ correspondem a $82,09 \%$ dos indivíduos inventariados. Para as classes de diâmetro inventariadas, as Cactaceae corresponderam a $0,48 \%$, sugerindo ser esta vegetação de caatinga de caráter xerófilo pouco acentuado. As 10 espécies mais importantes, determinadas pelo índice de valor de importância, foram Commiphora leptophloeos $(22,28)$, Croton zehntneri $(19,39)$, Mimosa sp. $(17,24)$, Tabebuia spongiosa $(16,99)$, Myracrodruon urundeuva $(15,38)$, Anadenanthera macrocarpa $(14,25)$, Coccoloba termiflora $(11,73)$, Caesalpinia sp. $(11,17)$, Cordia sp. $(10,79)$ e Tabebuia sp. $(10,20)$.
\end{abstract}

Palavras-chave - fitossociologia, vegetação, diversidade

ABSTRACT - (Floristic and fitosociological survey of a caatinga area at "Contendas do Sincora",
Bahia State, a homogeneous microregion of the Chapada Diamantina). The aim of this work was to
conduct a floristic and phytosociological survey on 11,034 hectares of the Extrema Lapinha Farm
$\left(13^{\circ} 46^{\prime}\right.$ to $14^{\circ} \mathrm{S}$ and $41^{\circ} 03^{\prime}$ to $41^{\circ} 10^{\prime} \mathrm{W}$ ), altitude from 295 to $380 \mathrm{~m}$, in the town of "Contendas do
Sincora", in Chapada Diamantina, Bahia State. The climate is of the BSwh' type, hot semi-arid,
with an annual rainfall of $500-700 \mathrm{~mm}$, the rainy season being from November to January, with a
mean temperature of $23^{\circ} \mathrm{C}$, and a relative humidity of $60-80 \%$. The soils are podzolic red-yellow
eutrophic and eutrophic litholic, with a lightly wavy relief. One hundred $20 x 5 \mathrm{~m}$ plots were randomly

1 Embrapa Semi-Árido, C. Postal 23, CEP 56300-000, Petrolina, PE, Brasil. e-mail: pcflima@cpatsa. embrapa.br; luciano@cpatsa.embrapa.br 
distributed, where all plants with a diameter at breast height equal to or greater than $5 \mathrm{~cm}$ were recorded. A total of 2,897 specimens was found, comprising 71 species, 51 genera and 23 botanic families with a $3.59 \%$ of them not identified. The families Euphorbiaceae $(15.67 \%)$, Mimosaceae $(13.80 \%)$, Bignoniaceae $(12.35 \%)$, Caesalpiniaceae $(7.14 \%)$, Boraginaceae $(6.63 \%)$, Polygonaceae $(6.63 \%)$, Anacardiaceae $(6.04 \%)$, Burseraceae $(4.93 \%)$, Apocynaceae $(4.59 \%)$, and Combretaceae $(4.28 \%$ ) comprehended $82.09 \%$ of the surveyed specimens. The Cactaceae family represented $0.48 \%$ of the total number of this families, suggesting little evidence of a xerophylous characteristic in this caatinga site. The 10 most important species by the importance value index were: Commiphora leptophloeos (22.28), Croton zehntneri (19.39), Mimosa sp. (17.24), Tabebuia spongiosa (16.99), Myracrodruon urundeuva (15.38), Anadenanthera macrocarpa (14.25), Coccoloba termiflora (11.73), Caesalpinia sp. (11.17), Cordia sp. (10.79), and Tabebuia sp. (10.20).

Key words - plant sociology, vegetation, diversity

\section{Introdução}

Estudos da interação planta-comunidade têm sido realizados em seus aspectos morfo-biológico e sinecológico, com objetivo de descrição florística e classificação fisionômica e ecológica. No nordeste, diversos trabalhos vem sendo realizados baseados na fitogeografia, associação de gêneros e de espécies dominantes. Duque (1964), utilizando aspectos fitogeográficos regionais, dividiu-a em Caatinga stricto sensu, Sertão, Seridó, Cariris Velho, Curimataú, Carrasco, Serras e Agreste. Ainda, buscando classificação através de informações de solo, índice xerotérmico, fisionomia e associação dos gêneros e das espécies, Andrade-Lima (1981) agrupou os 12 tipos de associações que encontrou em seis grandes unidades ecológicas.

Quanto aos aspectos sinecológicos, tem-se observado que a precipitação é o fator ambiental condicionante dos diferentes padrões de caatinga. Em geral, a similaridade entre vegetação varia das comunidades de menor densidade e maior porte, para aquelas de maior densidade e menor porte. Drumond et al. (1982), em Santa Maria da Boa Vista, PE, constataram que, dentre as espécies de caatinga naquela região, Mimosa hostilis (Mart.) Benth. (jurema-preta) foi a espécie responsável pela similaridade entre as parcelas inventariadas.

Ferraz et al. (1998), na análise da similaridade florística entre áreas de caatinga e brejos de altitude no Vale do Pajeú, PE, encontraram do total de 159 espécies, 23 comuns e exclusivas das altitudes de 1.100 e $900 \mathrm{~m}$, e um outro grupo constituído por espécies de caatinga das áreas de 700 e $500 \mathrm{~m}$.

Em estudo de remanescente de caatinga à margem do Rio São Francisco, Petrolina, PE, Nascimento (1998) encontrou dois conjuntos florísticos formados por espécies de Mimosa bimucronata Kunth e Geoffroea spinosa Jacq. ligados a ambientes topográficos do terraço fluvial, e outro por Mimosa tenuiflora (Wild.) Poir., Aspidosperma pyrifolium Mart., Caesalpinia microphylla Mart., Commiphora leptophloeos (Mart.) J. B. Gillett, Schinopsis brasiliensis Engl., relacionados ao tabuleiro sertanejo.

Sampaio (1996), em revisão sobre estudos de fitossociologia no nordeste, lista Caesalpinia pyramidalis Tul., Croton sonderianus Muell. Arg., Aspidosperma pyrifolium, Mimosa spp.(juremas- pretas), Jatropha spp. (pinhão), Croton spp. (quebrafaca), Commiphora leptophloeos, Anadenanthera columbrina var. cebil (Grisch.) Altschol., Schinopsis glabra (Engl.) F. A. Barkley \& T. Mey, Spondias tuberosa Arruda 
e Myracrodruon urundeuva como espécies de caatinga nas primeiras colocações em trabalhos relativos à densidade, área basal ou volume de madeira.

Quanto à florística de caatinga em geral, trabalhos têm sido realizados demonstrando ser a vegetação rica em cactáceas e bromeliáceas no estrato rasteiro, e no arbóreo dominam ecotipos endêmicos, como Spondias tuberosa (Anacardiaceae), Commiphora leptophloeos (= Bursera leptophloeos (Mart.) Engl. - Burseraceae), Cnidosculus phyllacanthus Pax. et K. Hoffm. (Euphorbiaceae), Aspidosperma pyrifolium (Apocynaceae) e outras. Como ochlospécies (espécies de ampla distribuição), são comuns Anadenanthera macrocarpa (Mimosaceae) e Myracrodruon urundeuva (Anacardiaceae). Barbosa et al. (1996) listam espécies presentes em levantamentos de biomas do nordeste, chegando ao total de cerca de sete mil espécies.

Nas zonas de ação antrópica, estudos têm sido realizados a fim de quantificar se as formas de manejo tem alterado a estrutura e similaridade da vegetação. Objetiva-se neste trabalho retratar em seus aspectos fitossociológicos, uma área de vegetação natural de caatinga, na microrregião da Chapada Diamantina. A análise da abundância, freqüência, dominância, posição sociológica das espécies inventariadas, bem como outros tópicos da estrutura da vegetação, servirão de subsídios para planos de manejo na referida área.

\section{Material e métodos}

O trabalho foi realizado na Fazenda Extrema-Lapinha, Contendas do Sincorá, Bahia, área de 11.000 ha, pertencente à Magnesita SA, em 1990. A região enquadra-se em clima semi-árido quente (tipo BSwh' pela classificação de Köppen), com estação chuvosa no período de novembro a janeiro, precipitação entre 500 a $1.000 \mathrm{~mm}$ anuais, temperatura de 21 a $28^{\circ} \mathrm{C}$ e umidade relativa de 60 a $70 \%$. Predominam os solos Podzólicos Vermelho Amarelo Equivalente Eutrófico e solos Litólicos Eutróficos.

Foram distribuídas aleatoriamente, 100 parcelas retangulares de $20 \times 5 \mathrm{~m}$ para levantamento das espécies. Árvores com DAP $\geq 5,0 \mathrm{~cm}$ foram inventariadas, tendo o material botânico coletado recebido número de identificação da árvore, nome vulgar e em seguida, herborizados, e incorporados ao Herbário do Trópico Semi-Árido (TSAH).

O tamanho da parcela foi determinado em função de trabalhos realizados pela EMBRAPA (1979) sobre dimensões requeridas em levantamentos fitossociológicos em vegetação de caatinga, onde o tamanho mínimo é de $64 \mathrm{~m}^{2}$. Os critérios usados na determinação da abundância, freqüência, dominância, índice de valor de importância, valor de cobertura e posição sociológica das espécies estão descritas em MuellerDombois \& Ellemberg (1974), Rosot et al. (1982), Hosokawa (1986) e Rodal et al. (1992) . Os limites das classes de altura para o estrato superior, médio e inferior da vegetação com árvores com $\mathrm{DAP} \leq 5,0 \mathrm{~cm}$ foram determinados graficamente pela divisão eqüitativa da porcentagem de freqüência acumulada da altura das mesmas.

\section{Resultados e discussão}

A relação das espécies inventariadas está na Tab.1. Existe predominância de caatinga arbórea com presença de espécies da mata seca e cerrado, demonstrando ser 
esta uma região de transição dos biomas caatinga, cerrado e mata, que interpenetramse, tornando-se difícil estabelecer limites. De acordo com descrição de unidades de caatinga apresentada por Andrade-Lima (1981), esta vegetação corresponde à Catinga de Floresta Alta (vegetação tipo 1: Tabebuia-Aspidosperma-Astronium-Cavanillesia), onde gêneros e espécies como Myracrodruon urundeuva, Schinopsis brasiliensis, Tabebuia sp., Aspidosperma pyrifolium e Cereus jamacaru são freqüentes. De acordo com este autor, este tipo de vegetação é comum no norte de Minas Gerais e sudoeste central da Bahia, que inclui o município ora estudado. Algumas árvores típicas das matas secas podem coabitar o cerrado e vice-versa (Heringer et al. 1976).

Tabela 1 - Relação das espécies inventariadas na fazenda Extrema-Lapinha, em Contendas do Sincorá, BA

\begin{tabular}{|c|c|c|}
\hline Nome vulgar & Nome Científico & Família \\
\hline Amargoso & Aspidosperma cylindrocarpon Muell. Arg. & Apocynaceae \\
\hline Amendoim & Pterogyne nitens Tul. & Caesalpiniaceac \\
\hline Angico & Anadenanthera macrocarpa (Benth.) Brenan & Mimosaceae \\
\hline Angiquinho & Sesbania sp. & Fabaceae \\
\hline Araticum & Annona glabra L. & Annonaceae \\
\hline Aroeira & Myracrodruon urundeuva FF. \& MF. Allemão & Anacardiaceae \\
\hline Braúna & Schinopsis brasiliensis Engl. & Anacardiaceae \\
\hline Barriguda & Canavillesia hylogeiton Urb. & Bombacaceae \\
\hline Bocaiúva & Não identificada 2 & \\
\hline Cagaita & Eugenia dysenterica DC. & Myrtaceae \\
\hline Cambuim & Eugenia crenata Vell. & Myrtaceae \\
\hline Canjoão & Senna spectabilis (DC.) Irwin \& Barneby & Caesalpiniaceae \\
\hline Canjoãozinho & $S$ catingae Harms & Caesalpiniaceae \\
\hline Canjerana/Cedro & Trichilia sp. & Meliaceae \\
\hline Cansanção & Cnidoscolus bahianus (Ule) Pax \& K. Hoffm. & Euphorbiaceae \\
\hline Caraiba & Tabebuia caraiba (Mart.) Burret & Bignoniaceae \\
\hline Casca fina & Fraunhofera multiflora Mart. & Celastraceae \\
\hline Casquinha & Senna sp. & Caesalpiniaceae \\
\hline Catinga de porco & Croton zehntneri Pax \& K. Hoffm. & Euphorbiaceae \\
\hline Cipó boi & Arrabidea sp. & Bignoniaceae \\
\hline Cola prato & Não identificada 3 & \\
\hline Cruzeta & Chomelia sp. & Rubiaceae \\
\hline Esporão de galo & Cordia sp. & Boraginaceae \\
\hline Farinha seca & Pisonia tomentosa Casar. & Nyctaginaceae \\
\hline Faveira & Caesalpinia microphylla Mart. & Caesalpiniaceae \\
\hline Folha larga & Tabebuia sp. & Bignoniaceae \\
\hline Folha miúda & Caesalpinia sp. & Caesalpiniaceae \\
\hline Guariroba & Eugenia sp. & Myrtaceae \\
\hline Imbiruçu & Pseudobombax simplicifolium A. Robyns & Bombacaceae \\
\hline Imburaé & Não identificada 1 & \\
\hline Incó & Capparis yco Eichl. & Capparidaceae \\
\hline Juazeiro & Ziziphus joazeiro Mart. & Rhamnaceae \\
\hline Jurema & Mimosa sp. & Mimosaceae \\
\hline Jureminha & Acacia piauhiensis Benth. & Mimosaceae \\
\hline Lava cabeça & Poeppigia procera Presl. & Caesalpiniaceae \\
\hline Leiteiro & Sapium montevidense Klotzsch ex Baill. & Euphorbiaceae \\
\hline Limãozinho & Fagara sp. & Rutaceae \\
\hline Madeira de curral & Terminalia fagifolia Mart. \& Zucc. var. parvifolia & Combretaceae \\
\hline Mandacaru & Cereus jamaracu DC. & Cactaceae \\
\hline Mandioca brava & Manihot pseudoglasiovii Pax \& K. Hoffm. & Euphorbiaceae \\
\hline
\end{tabular}


Tabela 1. Relação das espécies inventariadas na fazenda Extrema-Lapinha, em Contendas do Sincorá, BA (continuação)

\begin{tabular}{|c|c|c|}
\hline Nome vulgar & Nome Científico & Familia \\
\hline Mão zê & Caesalpinia sp. & Caesalpiniaceae \\
\hline Mulungu & Erythrina velutina Willd. & Fabaceae \\
\hline Pau d'arco & Tabebuia impetiginosa (Mart. DC.) Standl. & Bignoniaceae \\
\hline Pau de colher & Maytenus rigida Mart. & Celastraceae \\
\hline Pau mocó & Triplaris pachau Mart. & Polygonaceae \\
\hline Pau sangue & $\begin{array}{l}\text { Pterocarpus violaceus } \text { Vog. } \\
\text { var. angustifolia } \text { Benth. }\end{array}$ & Fabaceae \\
\hline Pau vidro & Aspidosperma $\mathrm{sp}$. & Apocynaceae \\
\hline Pereira & Aspidosperma refractum Mart. & Apocynaceae \\
\hline Pimentinha & Xilopia aromatica (Lam.) Mart. & Annonaceae \\
\hline Pinhão & Jatropha molissima Pohl & Euphorbiaceae \\
\hline Pratudo & Sweetia cf. dasycarpa & Fabaceae \\
\hline Putumuju & Poecilanthe subcordata Benth. & Fabaceae \\
\hline Quaçu & Coccoloba termiflora Lind. & Polygonaceae \\
\hline Quiabento & Pereskia grandifolia Hancort & Cactaceae \\
\hline Racha-racha & Pithecellobium sp. & Mimosaceae \\
\hline Rama de garrote & Cordia sp. & Boraginaceae \\
\hline Sete cascas & Tabebuia spongiosa Rizzini & Bignoniaceae \\
\hline Sucupira & Pterodon abruptus ( Moric. ) Benth. & Fabaceae \\
\hline Taipoca/Xixá & Cordia sp. & Boraginaceae \\
\hline Tamarindo & Senna cana Mart. & Caesalpiniaceae \\
\hline Tamboril & Peltophorum dubium (Spreng.) Taub. & Caesalpiniaceae \\
\hline Tapicuru & Goniorrhachis marginata Taub. & Caesalpiniaceae \\
\hline Tatarema & Pithecellobium parvifolium (Willd.) Benth. & Mimosaceae \\
\hline Tatu & Esembeckia aff. mollis Miq. & Rutaceae \\
\hline Tingui & Magonia glabrata St. Hill & Sapindaceae \\
\hline Umburana & Bursera leptophloeos (Mart.) Engl. & Burseraceae \\
\hline Umburana macho & Amburana cearensis (Fr. All.) A. C. Smith & Fabaceae \\
\hline Umbuzeiro & Spondias tuberosa Arruda & Anacardiaceae \\
\hline Unha de gato & Acacia paniculata Willd. & Mimosaceae \\
\hline Vaqueta & Thiloa glaucocarpa (Mart.) Eichl. & Combretaceae \\
\hline Velame & Croton argyrophylloides Muell. Arg. & Euphorbiaceae \\
\hline
\end{tabular}

Quanto a espécies de cerrado, da totalidade inventariada, Terminalia fagifolia, Eugenia dysenterica, Magonia glabrata, Tabebuia caraiba e Xylopia aromatica são comumente encontradas no cerrado mineiro (Brandão \& Gavilanes 1992). As espécies Myracrodruon urundeuva, Tabebuia caraiba, Eugenia dysenterica e Terminalia fagifolia também são comuns aos cerrados do Piauí, Maranhão e Planalto Central do Brasil (Heringer et al. 1971). Segundo Rizzini (1997), embora a flora do cerrado comum seja heterogênea, o que a torna profundamente divergente da caatinga é a constituição da flora exclusiva. $\mathrm{Na}$ análise de similaridade entre a vegetação de Serra Velha, em Padre Marcos, PI, Oliveira et al. (1997) encontraram baixo índice com os cerrados nordestinos, com apenas nove espécies em comum.

Dentre as espécies das zonas de mata seca do nordeste, foram encontradas Aspidosperma cylindrocarpon, A. refractoum, Cavanillesia hylogeiton, Pterocarpus violaceu e Chomelia sp. As presenças de Myracrodruon urundeuva e Anadenanthera macrocarpa no levantamento confirmam a ampla distribuição das mesmas em diferentes ecossistemas do Brasil. 
Quanto à composição florística, foram inventariadas 2.897 árvores, abrangendo 71 espécies, 51 gêneros e 23 famílias botânicas, conforme dados apresentados na Tab. 2. Não foram identificadas três espécies, correspondendo a 3,59\% dos indivíduos encontrados. Para efeito de cálculos da composição florística eles foram incluídos em um só gênero e família.

Tabela 2. Distribuição do número de espécies, gênero e famílias, e porcentagem de indivíduos inventariados na fazenda Extrema-Lapinha, em Contendas do Sincorá, BA.

\begin{tabular}{lcccc}
\hline Família & Gêneros & Espécies & $\begin{array}{c}\text { Número } \\
\text { indiv./ha }\end{array}$ & $\begin{array}{c}\text { \% sobre o } \\
\text { total }\end{array}$ \\
\hline Euphorbiaceae & 5 & 6 & 454 & 15,67 \\
Mimosaceae & 4 & 6 & 400 & 13,80 \\
Bignoniaceae & 2 & 5 & 358 & 12,35 \\
Caesalpiniaceae & 6 & 11 & 207 & 7,17 \\
Polygonaceae & 2 & 2 & 192 & 6,63 \\
Boraginaceae & 1 & 3 & 192 & 6,63 \\
Anacardiaceae & 3 & 3 & 175 & 6,04 \\
Burseraceae & 1 & 1 & 143 & 4,93 \\
Apocynaceae & 1 & 3 & 133 & 4,59 \\
Combretaceae & 2 & 2 & 124 & 4,28 \\
Annonaceae & 2 & 2 & 72 & 2,49 \\
Celastraceae & 2 & 2 & 58 & 2,00 \\
Bombacaceae & 2 & 2 & 54 & 1,86 \\
Fabaceae & 7 & 7 & 53 & 1,82 \\
Myrtaceae & 1 & 3 & 51 & 1,76 \\
Meliaceae & 1 & 1 & 42 & 1,45 \\
Nyctaginaceae & 1 & 1 & 34 & 1,17 \\
Capparidaceae & 1 & 1 & 21 & 0,73 \\
Cactaceae & 2 & 2 & 14 & 0,48 \\
Rutaceae & 2 & 2 & 8 & 0,28 \\
Sapindaceae & 1 & 1 & 4 & 0,15 \\
Rubiaceae & 1 & 1 & 2 & 0,08 \\
Rhamnaceae & 1 & 1 & 2 & 0,08 \\
Não identificada & - & 3 & 104 & 3,59 \\
\hline TOTAL & 51 & 71 & 2897 & 100 \\
\hline & & & & \\
\hline & & & 53 & \\
\hline
\end{tabular}

O número de espécies encontrado foi superior aos apresentados por Lima et al. (1997a; 1997b) em Ouricuri e Afrânio, PE, onde foram levantados, respectivamente, 2.936 indivíduos ( 45 espécies, 36 gêneros e 19 famílias) e 1.603 indivíduos (42 espécies, 35 gêneros e 18 famílias). Entretanto, para o Vale do Pajeú, PE, em trechos de vegetação de caatinga e brejo de altitude, Ferraz et al. (1998) encontraram 159 espécies (101 gêneros e 45 famílias) de cipós, arbustos e árvores. No Vale do Moxotó, PE, Rodal (1984) encontrou 214 espécies distribuídas em 52 famílias. As diferenças no número de espécies encontradas nestes levantamentos podem estar centradas nas metodologias utilizadas, bem como em características de sítio.

A heterogeneidade florística da vegetação da Fazenda Extrema-Lapinha é elevada, tendo sido encontrada a média de dois gêneros por família. $O$ valor médio de 1:2 obtido nas parcelas inventariadas para o Quociente de Mistura de Jentsch (QMJ) significa que a cada $100 \mathrm{~m}^{2}$ de caatinga encontrou-se, em média, duas árvores por espécie. Logo, os 
valores encontrados revelam grande heterogeneidade florística da vegetação, sendo que as Euphorbiaceae, Mimosaceae, Bignoniaceae, Caesalpiniaceae, Boraginaceae, Polygonaceae, Anacardiaceae, Burseraceae, Combretaceae e Apocynaceae correspondem a $82,09 \%$ dos indivíduos inventariados. Vale ressaltar que, em levantamentos da caatinga, estas famílias são as mais freqüentes e que apresentam maior número de indivíduos por hectare (Alcoforado Filho 1993; Ferraz et al. 1998)

No presente levantamento, as espécies dominantes do povoamento foram Commiphora leptophloeos $\left(4,717 \mathrm{~m}^{2} / \mathrm{ha}\right)$, Myracrodruon urundeuva $\left(2,571 \mathrm{~m}^{2} / \mathrm{ha}\right)$, Anadenanthera macrocarpa $\left(2,396 \mathrm{~m}^{2} / \mathrm{ha}\right)$, Tabebuia spongiosa $\left(2,030 \mathrm{~m}^{2} / \mathrm{ha}\right) \mathrm{e}$ Aspidosperma cylindrocarpon $\left(1,765 \mathrm{~m}^{2} / \mathrm{ha}\right)$, embora as maiores freqüências e abundâncias tenham sido para Mimosa sp. (75\%, 222 ind./ha) e Croton zehntneri (73\%, 308 ind./ha). Como a visão mais abrangente da estrutura da vegetação está nos valores do Índice de Valor de Importância (IVI), os maiores índices foram para Commiphora leptophloeos (22,28), Croton zehntneri (19,39), Mimosa sp.(17,24), Tabebuia spongiosa $(16,99)$, Myracrodruon urundeuva $(15,38)$, Anadenanthera macrocarpa $(14,25)$, Coccoloba termiflora (11,73), Caesalpinia sp. (11,17), Cordia sp. $(10,79)$, Tabebuia sp. $(10,20)$, Aspidosperma cylindrocarpon $(10,12)$, Triplaris pachau $(10,08)$, não identificada 1 (imburaé) $(8,53)$ e Schinopisis brasiliensis $(8,17)$. Os valores de freqüência, abundância e dominância, IVI e VC (Valor de Cobertura) das 18 principais espécies, correspondendo a $25 \%$ das inventariadas, estão na Tab. 3.

Quanto ao VC, estas mesmas espécies também surgem como principais, sendo que o maior índice foi para Commiphora leptophloeos $(17,94)$ seguida de Croton zehntneri $(14,11)$, com alterações nas ordens de classificações entre Tabebuia spongiosa $(12,21)$ e Mimosa sp. $(11,81)$ na composição florística da área, entre os dez primeiros colocados.

Quanto à estrutura vertical, o estrato médio da vegetação ficou definido para as árvores e arbustos compreendidos entre as alturas de 3,75 e 5,15m. No estrato inferior foram encontrados 643 árvores com DAP $\geq 5,0 \mathrm{~cm}$, pertencentes a 57 espécies, correspondendo a $22,2 \%$ dos indivíduos inventariados. Nos estratos médio e superior foram encontrados 61 e 62 espécies, correspondendo a 42,9 e 34,9\% do total das árvores inventariadas, respectivamente.

Na Tab. 4 estão os valores referentes aos índices da estrutura vertical das 18 principais espécies encontradas na vegetação. Croton zehntneri foi a espécie que apresentou a maior Posição Sociológica (PS), seguida de Mimosa sp. e Tabebuia spongiosa. Entretanto, estas duas últimas foram as que apresentaram valores mais regulares quanto ao número de indivíduos em todos os estratos. Uma espécie tem seu lugar assegurado na estrutura e composição da vegetação quando é representada em todos os estratos, principalmente em maior número no inferior, expressando o processo natural de regeneração. Dentre as 71 espécies inventariadas, 45 encontram-se presentes nos três estratos, sendo que Goniorrhachis marginata, Senna spectabilis, S. catingae, Fraunhofera multiflora, Cordia sp. (Esporão de galo), Euginia sp. (Guariroba), Ziziphus joazeiro, Sweetia cf. dasycarpa, Poecilanthe subcordata, Pterodon abruptus, Peltophorum dubium, Acacia paniculata e a espécie não identificada 2 (Bocaiúva) não apresentaram indivíduos no estrato inferior. 


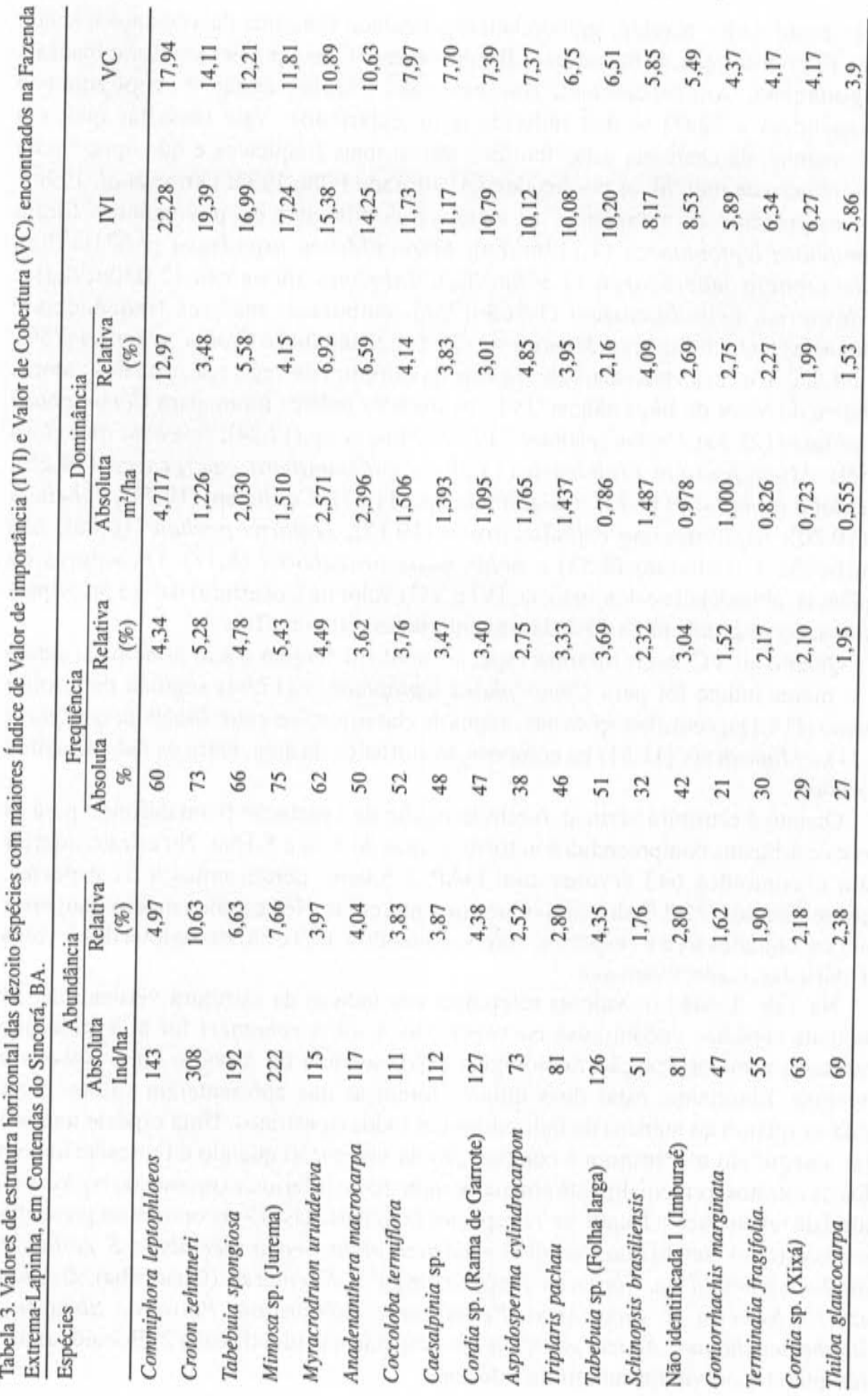




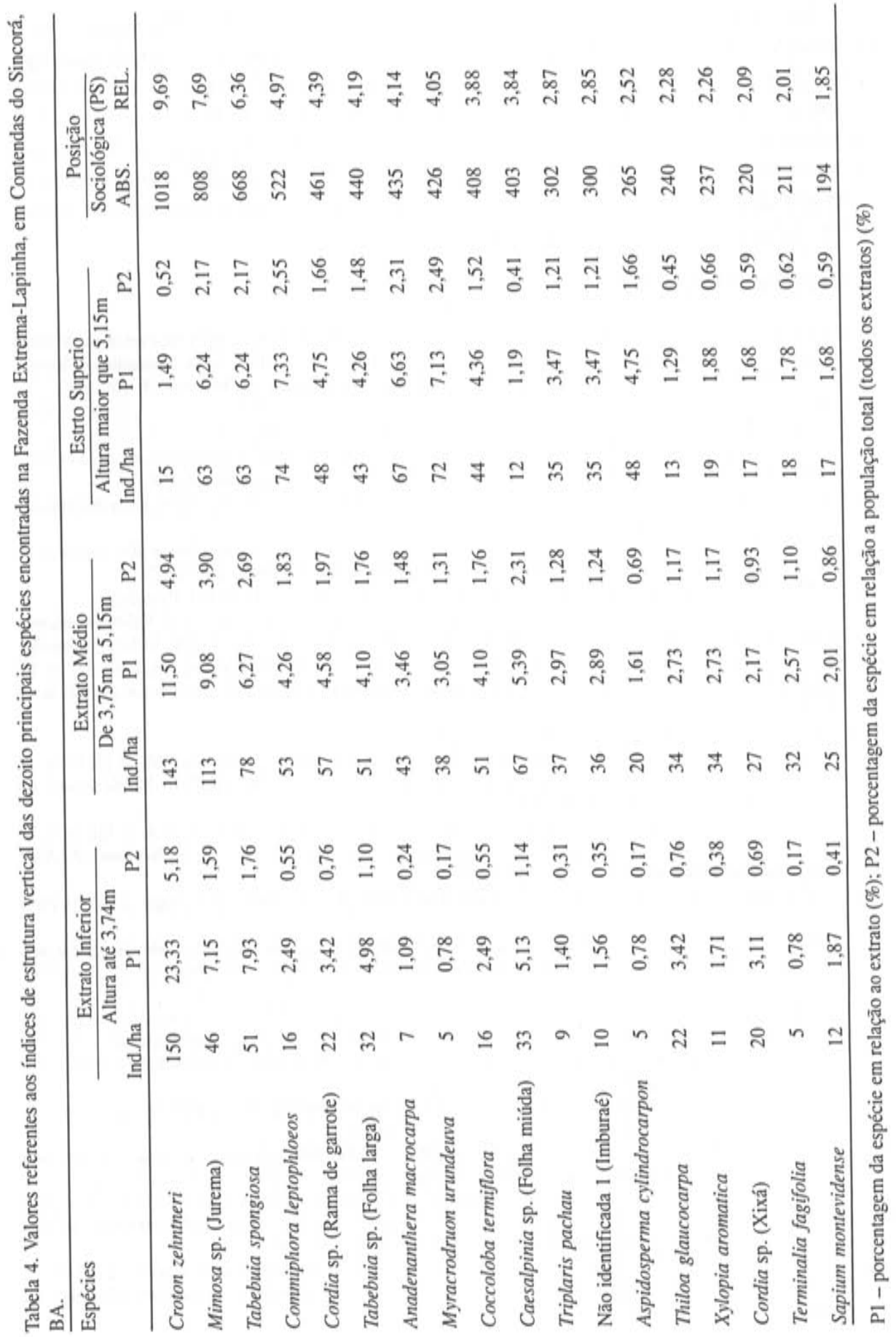


Em geral, as espécies listadas com maior IVI, VC e PS, expressando as estruturas horizontal e vertical da população, corresponderam às espécies típicas de caatinga, como Croton zehntneri, Mimosa sp., Tabebuia spongiosa e Commiphora leptophloeos. Dentre as que ocorrem em cerrado, destaque para Terminalia fagifolia listada entre as 26 principais espécies da estrutura, correspondendo a um terço da vegetação. Constatouse baixa freqüência de cactaceas com DAP $>5 \mathrm{~cm}$, comuns em levantamentos de vegetação de caatinga com características hiperxeromórficas, sendo inventariadas apenas Cereus jamacaru e Pereskia grandifolia.

\section{Referências bibliográficas}

Alcoforado Filho, F. G. 1993 Composição florística e fitossociológica de uma área de caatinga arbórea no município de Caruaru, PE. Dissertação de Mestrado. Universidade Federal de Pernambuco, Recife.

Andrade-Lima, D. de. 1981. The caatingas dominium. Revista Brasileira de Botânica 4: 149-163.

Barbosa, M. R. de ; Mayo, S. J. ; Castro, A. A. J. F. de; Freitas, G. L. de; Pereira, M. do S.; Gadelha Neto, P. da C. \& Moreira H. M. 1996. Checklist preliminar das angiospermas. Pp. 253-415 In: E. V. S. B. Sampaio; S. J. Mayo \& M. R. V. Barbosa (Eds.), Pesquisa botânica nordestina: progresso e perspectivas. Sociedade Botânica do Brasil, Seção Regional de Pernambuco, Recife.

Brandão, M. \& Gavilanes, M. L. 1992. Espécies arbóreas padronizadoras do cerrado mineiro e sua distribuição no estado. Informe Agropecuário 16 (173): 5-11.

Drumond, M. A; Lima, P. C. F.; Souza, S. M. de \& Lima, J. L. S. 1982. Sociabilidade das espécies da caatinga em Santa Maria da Boa Vista-PE. Boletim de Pesquisa Florestal (4): 47-59.

Duque, G. 1964. O nordeste e as lavouras xerófilas. Banco do Nordeste do Brasil, Fortaleza.

EMBRAPA. Centro de Pesquisa Agropecuário do Trópico Semi-Árido, Petrolina-PE. 1979. Relatório técnico anual do Centro de Pesquisa Agropecuário do Trópico Semi-Árido, 1997-1998. EMBRAPA-DID, Brasília.

Heringer, E. P.; Barroso, G. M.; Rizzo, J. A. \& Rizzini, C. T. 1977. A flora do cerrado. Pp. 211-232. In: IV Simpósio sobre o cerrado: bases para utilização agropecuária. Ed. Itatiaia, Belo Horizonte e Editora da Universidade de São Paulo, São Paulo.

Hosokawa, R. T. 1986. Manejo e economia de florestas. FAO, Roma.

Lima, J. L. S. de; Cavalcanti, N. de B.; Lima, E. R. de ; Carvalho, K. M. de ; Oresotu, B.A. \& Oliveira, C. A. V. 1997a. Levantamento fitoecológico do município de Afrânio, PE. Pp. 230. In: Resumos do
XLVIII Congresso Nacional de Botânica. Fortaleza:BNB.

Lima, J. L. S. de; Cavalcanti, N. de B.; Lima, E. R. de ; Carvalho, K. M. de ; Oresotu, B.A. \& Oliveira, C. A. V. 1997b. Levantamento fitoecológico do município de Ouricuri, PE. Pp. 230. In: Resumos XLVIII Congresso Nacional de Botânica. Fortaleza:BNB. Mueller-Dombois, D. \& Ellemberg, H. 1974. Aims and methods of vegetation ecology. J.Wiley, New
York.

Nascimento, C. E. de S. 1998. Estudo florístico e fitossociológico de um remanescente de caatinga à margem do Rio São Francisco, Petrolina, Pernambuco. Dissertação de Mestrado. Universidade
Federal Rural de Pernambuco, Recife.

Oliveira, M. E. A. de ; Sampaio, E. V. S. B.; Castro, A. A. J. F. \& Rodal, M. J. N. 1997. Flora e fitossociologia de uma área de transição carrasco-caatinga de areia em Padre Marcos, Piauí. Naturalia 22: 131-150.

Rizzini, C. T. 1997. Tratado de fitogeografia do Brasil: aspectos ecológicos, sociológicos e florísticos. Âmbito Cultural Edições, Rio de Janeiro.

Rodal, M. J. N. 1984. Fitoecologia de uma área do médio Vale do Moxotó, Pernambuco. Dissertação de Mestrado. Universidade Federal Rural de Pernambuco, Recife.

Rodal, M. J. N.; Sampaio, E. V. S. B. \& Figueiredo, M. A. 1992. Manual sobre métodos de estudos florísticos e fitossociológicos - ecossistema caatinga. Sociedade Botânica do Brasil, Brasília. Rosot, N. C.; Machado, S. do A. \& Figueiredo Filho, A. 1982. Análise estrutural de uma floresta tropical
como subsídio básico para elaboração de um plano de manejo florestal. In: Anais do Congresso Nacional sobre Essências Nativas. Silvicultura em São Paulo 16(A): 468-490.

Sampaio, E. V. S. B. 1996. Fitossociologia. Pp.203-224. In: E. V. S. B. Sampaio; S. J. Mayo \& M. R. V. Barbosa (Eds.), Pesquisa botânica nordestina: progresso e perspectivas. Sociedade Botânica do Brasil-Seção Regional de Pernambuco, Recife. 